\title{
Long-Term Clinical and Echocardiographic Outcome in Patients with CRT and Functional Mitral Regurgitation: MitraClip versus Optimal Medical Therapy
}

\author{
Arianna Cirasa, MD*, Antonio Popolo Rubbio, MD, Paolo Zappulla, MD, Angelo Di Grazia, MD, \\ Daniela Dugo, MD, Salvatore Scandura, MD, Sarah Mangiafico, MD, Carmelo Grasso, MD, Corrado \\ Tamburino, MD, PhD, Davide Capodanno, MD, PhD and Valeria Calvi, MD, PhD
}

\author{
Division of Cardiology, Centro Alte Specialità e Trapianti (CAST), Azienda Ospedaliero-Universitaria \\ Policlinico-Vittorio Emanuele, University of Catania, Italy
}

*Corresponding author: Arianna Cirasa, Division of Cardiology, Centro Alte Specialità e Trapianti (CAST), Azienda Ospedaliero-Universitaria Policlinico-Vittorio Emanuele, University of Catania, Via Santa Sofia 78 , 95123, Catania, Italy, Tel: +39-095-3781172, Fax: +39-095-3782742

\section{Abstract}

Objectives: This study sought to evaluate the clinical outcome in cardiac resynchronization therapy (CRT) patients with residual moderate-to-severe functional mitral regurgitation (FMR) who underwent percutaneous treatment with the Mitraclip System or were managed with medical therapy alone.

Background: Significant FMR can persist in $20-25 \%$ of patients after CRT and its management can be a challenge, due to the advanced heart failure (HF) and a variety of concomitant comorbidities.

Methods: From a single center, two different groups of patients with CRT (MitraClip and no-MitraClip group) were compared. Clinical follow-up was scheduled at 30-day, 12 months and 24 months in all patients. The primary endpoint was the composite of all-cause death or HF hospitalizations at 24-month follow-up.

Results: Forty patients (56\%) were treated with the Mitraclip System (MitraClip group) and 31 patients (44\%) only with medical therapy (no-MitraClip group). MitraClip group presented higher surgical risk than no-MitraClip group, as older age, chronic kidney disease, higher NYHA functional class and lower left ventricular ejection fraction. At 24-month no differences in all-cause mortality were detected between the two groups (Kaplan-Meier curves log-rank $p=0.947$ ), although the MitraClip group presented lower rate of HF hospitalizations than the no-MitraClip group (log-rank $p=$ 0.048). After adjustment for confounding factors, time to HF hospitalization or death was significantly delayed in MitraClip group (hazard ratio: 0.38; p-value 0.018).
\end{abstract}

Conclusion: In CRT patients, the treatment of moderateto-severe FMR with the Mitraclip System - if compared to medical therapy alone - is a safe and effective approach in reducing cardiac symptoms and the rate of hospitalizations for HF.

\section{Condensed Abstract}

From a single center, 71 patients with CRT and residual moderate-to-severe functional mitral regurgitation were treated with Mitraclip or with medical therapy alone.

At 24-month MitraClip patients presented lower rate of hospitalizations for heart failure (HF) than patients treated with only medical therapy. After adjustment for confounding factors, time to HF or death was significantly delayed in MitraClip group.

\section{Keywords}

Heart failure, Functional mitral regurgitation, Cardiac resynchronization therapy, Mitraclip

\section{Introduction}

Cardiac resynchronization therapy (CRT) is a recommended therapy for selected patients with systolic heart failure (HF) and intraventricular conduction delay [1]. Moderate-to-severe functional mitral regurgitation (FMR) occurs in approximately one-third of patients with HF referred to CRT and, although CRT therapy has been established as a treatment option in this cohort, 
significant FMR may persist or getting worse in approximately half of patients [2,3]. Over time, the increasing severity of FMR could be associated with adverse left ventricular remodeling and unfavorable outcome [4]. Furthermore, due to the advanced HF and a variety of concomitant comorbidities, surgical approach to FMR is often denied and patients non-responders to CRT can be managed with medical therapy alone or with less invasive percutaneous approaches $[5,6]$.

Percutaneous mitral valve repair with the Mitraclip system (Abbott Vascular, Santa Clara, CA) has emerged as a feasible alternative to surgery for the treatment of symptomatic moderate-to-severe FMR [7]. The EVEREST trial and different registries showed safety, efficacy and favorable outcome of the MitraClip in selected patients. Recently, two different randomized trials studied the outcome of the MitraClip versus medical therapy alone in FMR, getting controversial results in light of the very different population recruited [8,9]. Particularly the American COAPT trial showed that transcatheter mitral valve repair compared to medical therapy alone in FMR obtained a lower rate of hospitalization for HF and allcause mortality within 24 months of follow-up.

The treatment of moderate-to-severe FMR in CRT non-responders is feasible and reasonably effective in reducing cardiac symptoms and determining favorable cardiac remodeling, but previous studies in this field are limited by the lack of a control group and do not provide sustained information on long-term outcome of these patients $[10,11]$.

Based on this issue, this study sought to evaluate the long-term outcome (i.e. 24-month) of CRT patients with moderate-to-severe FMR who underwent percutaneous treatment with the Mitraclip System or were managed with medical therapy alone.

\section{Methods}

\section{Patient population}

We retrospectively analyzed patients who were referred to our Institution (Ferrarotto Hospital, Policlinico-Vittorio Emanuele, Catania, Italy) from June 2000 to May 2017 for the management of reduced ejection fraction HF. The institutional review board approval was given under the condition that the patients' data were collected in an aggregate and anonymized form.

In keeping with the ESC guidelines, patients underwent CRT if they had 1) New York Heart Association (NYHA) functional class III or IV despite optimal medical therapy, 2) Left ventricular ejection fraction (LVEF) $\leq 35 \%$, and 3) QRS duration $\geq 130 \mathrm{~ms}$, with left bundle branch block morphology [1]. Among patients with CRT, we retrospectively selected those with moderate-to-severe residual FMR, who represent the population of the present study. All patients were prescribed guideline-directed medical therapy, including maximum-tolerated doses of beta-blockers, angiotensin converting enzyme inhibitors or angiotensin receptor blockers, spironolactone and furosemide where appropriate. Clinical outcomes were then compared between patients who underwent Mitraclip therapy and those who were managed with medical therapy alone.

\section{Endpoints and definitions}

Clinical endpoints were defined according to the Mitral Valve Academic Research Consortium (MVARC) criteria [12]. The primary outcome of interest was the composite of all-cause death or cardiac hospitalizations for HF at 24-month follow-up. Secondary outcomes were all-cause death, re-hospitalization for HF and NYHA class at 24-month. Echocardiographic endpoints were assessed at 12-month, including parameters as LVEF, left ventricle end-diastolic and end-systolic volume, systolic pulmonary artery pressure and tricuspid regurgitation.

\section{Follow-up}

Follow-up data were obtained by scheduled clinical visits, echocardiographic assessment, and/or telephone calls at 30-day, 12-month and 24-month after the Mitraclip for the MitraClip group. The no-MitraClip group was studied in the same time window, as scheduled from the protocol of our Institute for patients with CRT. At each visit, the NYHA functional class was assessed. A standard echocardiographic examination was performed by two experienced echocardiographers, with FMR quantification as recommended by ASE/EAE guidelines $[13,14]$.

\section{Statistical analysis}

Categorical and dichotomous variables are presented as counts and percentages and were compared by chi-square or Fisher's exact tests, as appropriate. Continuous variables are presented as mean \pm standard deviation or median (interquartile range [IQR]) based on their distribution. Unpaired Student's t-tests were used to compare continuous parameters following a normal distribution, while Mann-Whitney $U$ tests were used to compare continuous variables with skewed distribution. Categorical and dichotomous variables are presented as counts and percentages and were compared by chisquare or Fisher's exact tests, as appropriate. A p value of 0.05 was set for significance.

A paired sample T-Test was used to evaluate clinical and echocardiographic outcomes in alive patients from baseline to follow up at 12-month.

Survival curves were obtained using the Kaplan Meier method, and were compared using the log-rank test. Multivariable Cox regression survival analyses were used to identify the relationship between the study population and the occurrence of adverse events (i.e. death or hospitalization) during the follow-up. For the multivariate Cox analysis, covariates entered in 
the model were considered for potential prognostic impact, on an epidemiological basis; these covariates included chronic kidney disease, NYHA class, male sex. For all tests, a two-tailed $p$-value $\leq 0.05$ was considered statistically significant. All data were processed using the Statistical Package for the Social Sciences version 21 (SPSS v21, Inc., Chicago, Illinois).

\section{Results}

\section{Patient population}

Of 71 patients with residual moderate-to-severe FMR in the study period, $40(56.4 \%)$ patients were treated with the Mitraclip System (MitraClip group) and $31(43.6 \%)$ patients were treated with medical therapy alone (no-MitraClip group) (Figure 1). Reasons for not performing the Mitraclip procedure in the 31 noMitraclip patients included: Refusal of the patient $(\mathrm{n}=$ $16 ; 51.6 \%)$, unfavorable anatomy ( $n=8 ; 25.8 \%)$, high risk of peri-procedural infection due to previous history of infection or vegetation on leads $(n=3 ; 9.7 \%)$ or death before the procedure $(n=4 ; 12.9 \%)$.

Baseline demographic characteristics of the study population and the MitraClip and no-MitraClip groups are summarized in Table 1 . The majority of patients were male $(69 \%)$ and the median age was 70 years (IQR 6476). Forty-seven patients (66\%) had idiopathic dilated cardiomyopathy and 36 (51\%) presented chronic kidney disease. The median NYHA Class was III (IQR II-III) and only 9 (13\%) patients were classified as CRT-responders. Criteria of CRT non-response were 1) No decrease of NYHA functional class; 2) Lack of an improvement of the left ventricular end-systolic volume of at least $10 \%$ after at least six months from the implantation of the device [15]. The median LVEF was 28\% (IQR 24-35\%). When comparing the two groups, the median patient age (MitraClip group 71.5 vs. no-MitraClip group 69 years, $p$-value 0.042 ) and the surgical risk score (STS mortality and morbidity, EuroSCORE II) were higher in the MitraClip group, as well as the proportion of male patients (MitraClip group $80 \%$ vs. no-MitraClip group $55 \%$, p-value 0.023), chronic kidney disease (MitraClip $65 \%$ vs. no-MitraClip $32 \%$, p-value 0.006 ), advanced NYHA class (III-IV) (MitraClip group $77.5 \%$ vs. noMitraClip group 22.5\%, p-value 0.000). In MitraClip group, LVEF was lower (MitraClip 25\% vs. no-MitraClip group $30 \%$, p-value 0.016 ) and left ventricle end-diastolic and end-systolic volumes were significantly larger than the no-MitraClip group. Patients in the no-MitraClip group significantly showed a higher prevalence of idiopathic dilated cardiomyopathy $(n=25 ; 80.6 \%)$.

\section{In-hospital and 30-day outcomes}

Peri-procedural and in hospital outcomes after the MitraClip are shown in Table 2. MVARC technical success was reported in 37 patients (92.5\%). The mean number of the implanted clips was 2 (IQR 1-2). Three peri-procedural complications were described: An episode of ventricular tachycardia interrupted by the intervention of the CRT-D; a laceration of the posterior leaflet of the mitral valve during the grasping maneuvers with consequent device failure; an iatrogenic fistula creation between the left atrium and the pulmonary artery, which was managed with a percutaneous occluder device. No in-hospital deaths were reported and the median length of stay was 4 days (IQR 2-6 days).

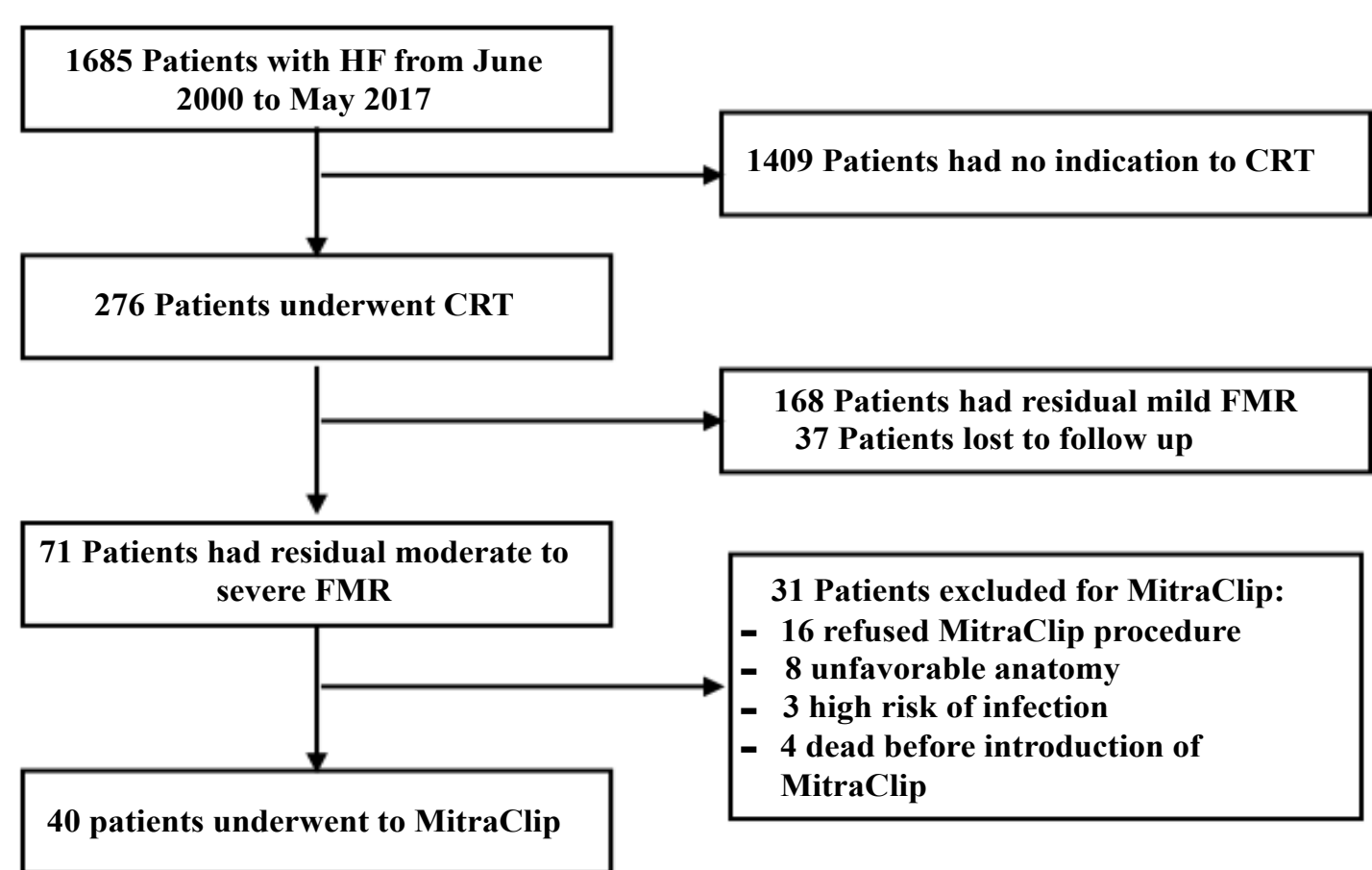

Figure 1: Flow Chart showing the patients included in the study. HF: Heart Failure; CRT: Cardiac Resynchronization Therapy; FMR: Functional Mitral Regurgitation. 
Table 1: Baseline demographic characteristics of the overall population.

\begin{tabular}{|c|c|c|c|c|}
\hline & Overall $(n=71)$ & MitraClip Group $(n=40)$ & No-MitraClip Group $(n=31)$ & $p$ value \\
\hline Age (years) & $70(64-76)$ & $71.5(67-77)$ & $69(59-72)$ & 0.042 \\
\hline Men, n (\%) & $49(69 \%)$ & $32(80 \%)$ & $17(54.8 \%)$ & 0.023 \\
\hline BMI $\left(\mathrm{Kg} / \mathrm{m}^{2}\right)$ & $26 \pm 3.6$ & $25.6 \pm 3.9$ & $26.5 \pm 3.1$ & 0.310 \\
\hline Idiopathic CMD, n (\%) & $47(66.2 \%)$ & $22(55 \%)$ & $25(80.6 \%)$ & 0.023 \\
\hline Hypertension, n (\%) & $52(73.2 \%)$ & $31(77.5 \%)$ & $21(67.7 \%)$ & 0.357 \\
\hline Diabetes, n (\%) & $16(22.5 \%)$ & $12(30 \%)$ & $4(12.9 \%)$ & 0.151 \\
\hline Dyslipidemia, n (\%) & $38(53.5 \%)$ & $20(50 \%)$ & $18(58.1 \%)$ & 0.499 \\
\hline Prior MI, n (\%) & $15(21.1 \%)$ & $11(27.5 \%)$ & $4(12.9 \%)$ & 0.156 \\
\hline Valve Surgery, n (\%) & $7(9.9 \%)$ & $4(10 \%)$ & $3(9.7 \%)$ & 1.000 \\
\hline Prior Stroke, n (\%) & $6(8.5 \%)$ & $3(7.5 \%)$ & $3(9.7 \%)$ & 1.000 \\
\hline $\mathrm{AF}, \mathrm{n}(\%)$ & $36(50.7 \%)$ & $19(47.5 \%)$ & $17(54.8 \%)$ & 0.540 \\
\hline COPD, n (\%) & $15(21.1 \%)$ & $8(20 \%)$ & $7(22.6 \%)$ & 0.792 \\
\hline Solid Tumours, n (\%) & $7(9.9 \%)$ & $3(7.5 \%)$ & $4(12.9 \%)$ & 0.691 \\
\hline CKD, n (\%) & $36(50.7 \%)$ & $26(65 \%)$ & $10(32.3 \%)$ & 0.006 \\
\hline Dialysis, n (\%) & $4(5.6 \%)$ & $2(5 \%)$ & $2(6.5 \%)$ & 1.000 \\
\hline GFR (mL/min) & $49(37-68)$ & $42(34-58)$ & $56(39-84)$ & 0.009 \\
\hline EuroSCORE II (\%) & $5.1(3.1-8.9)$ & $6(3.5-10.3)$ & $3.6(2.2-6.8)$ & 0.009 \\
\hline STS mortality (\%) & $2.2(1.2-5.0)$ & $3.7(1.7-6.4)$ & $1.3(0.75-2.4)$ & 0.000 \\
\hline STS morbidity (\%) & $22(16.1-31.5)$ & $27.3(19.7-42.1)$ & $16.7(12.1-23.8)$ & 0.000 \\
\hline NYHA Class & $3(2-3)$ & $3(3-3)$ & $2(2-3)$ & 0.000 \\
\hline NYHA III-IV, n (\%) & $40(56.3 \%)$ & $31(77.5 \%)$ & $9(22.5 \%)$ & 0.000 \\
\hline Responders CRT, n (\%) & $9(12.7 \%)$ & $6(15 \%)$ & $3(9.7 \%)$ & 0.504 \\
\hline \multicolumn{5}{|l|}{ Baseline Echo Variables } \\
\hline LVEF (\%) & $28(24-35)$ & $25(21-32)$ & $30(25-35)$ & 0.016 \\
\hline EDV-LV (ml) & $190(150-241)$ & $209(160-266)$ & $170(113-215)$ & 0.023 \\
\hline ESV-LV (ml) & $136(94-184)$ & $150(95-210)$ & $110(80-150)$ & 0.013 \\
\hline sPAP $(\mathrm{mmHg})$ & $45(40-60)$ & $50(41-60)$ & $40(36-60)$ & 0.089 \\
\hline Mild-Moderate TR, n (\%) & $59(83.1 \%)$ & $33(83.2 \%)$ & $26(83.9 \%)$ & 0.878 \\
\hline
\end{tabular}

Categorical variables are expressed as $n(\%)$. Continuous variables are expressed as mean \pm SD and in case of skewed distribution as median and IQR.

Abbreviations: BMI: Body Mass Index; CMD: Dilated Cardiomyopathy; MI: Myocardial Infarction; AF: Atrial Fibrillation; COPD: Chronic Obstructive Pulmonary Disease; CKD: Chronic Kidney Disease; GFR: Glomerular Filtration Rate; STS: Society of Thoracic Surgery; NYHA: New York Heart Association Functional Classification; LVEF: Left Ventricular Ejection Fraction; EDVLV: Left Ventricle End Diastolic Volume; ESV-LV: Left Ventricle End Systolic Volume; sPAPs: Systolic Pulmonary Artery Pressure; TR: Tricuspid Regurgitation.

Table 2: Procedural outcomes and in-hospital outcomes after MitraClip System.

\begin{tabular}{|l|l|}
\hline & MitraClip group (n = 40) \\
\hline Device time (min) & $49(29-75)$ \\
\hline Procedural time (min) & $119 \pm 42$ \\
\hline Implanted clips per patient (n) & $2(1-2)$ \\
\hline Technical success, $\mathrm{n}(\%)$ & $37(92.5 \%)$ \\
\hline MR grade 1+/2+ post implant, n (\%) & $37(92.5 \%)$ \\
\hline Post-procedural LVEF (\%) & $25(20-30)$ \\
\hline Post-procedural sPAP (mmHg) & $37(33-50)$ \\
\hline Periprocedural complication, $\mathrm{n}(\%)$ & $3(7.5 \%)$ \\
\hline Post-procedural Intubation, $\mathrm{n}(\%)$ bleeding, $\mathrm{n}(\%)$ & $0(0)$ \\
\hline Life threatening or Major MVARC & $1(2.5 \%)$ \\
\hline Minor MVARC bleeding, $\mathrm{n}(\%)$ & $2(5 \%)$ \\
\hline
\end{tabular}




\begin{tabular}{|l|l|}
\hline Major access and vascular complications, $\mathrm{n}(\%)$ & $1(2.5 \%)$ \\
\hline Minor access and vascular complications, $\mathrm{n}(\%)$ & $1(2.5 \%)$ \\
\hline Blood transfusion, $\mathrm{n}(\%)$ & $1(2.5 \%)$ \\
\hline AKI, $\mathrm{n}(\%)$ & $0(0)$ \\
\hline Stroke, $\mathrm{n}(\%)$ & $0(0)$ \\
\hline In-hospital death, $\mathrm{n}(\%)$ & $0(0)$ \\
\hline Length of stay (days) & $4(2-6)$ \\
\hline
\end{tabular}

Categorical variables are expressed as $\mathrm{n}(\%)$. Continuous variables are expressed as mean $\pm \mathrm{SD}$ and in case of skewed distribution as median and IQR.

Abbreviations: MR: Mitral Regurgitation; MVARC: Mitral Valve Academic Research Consortium; AKI: Acute Kidney Injury, Other abbreviations as in Table 1.

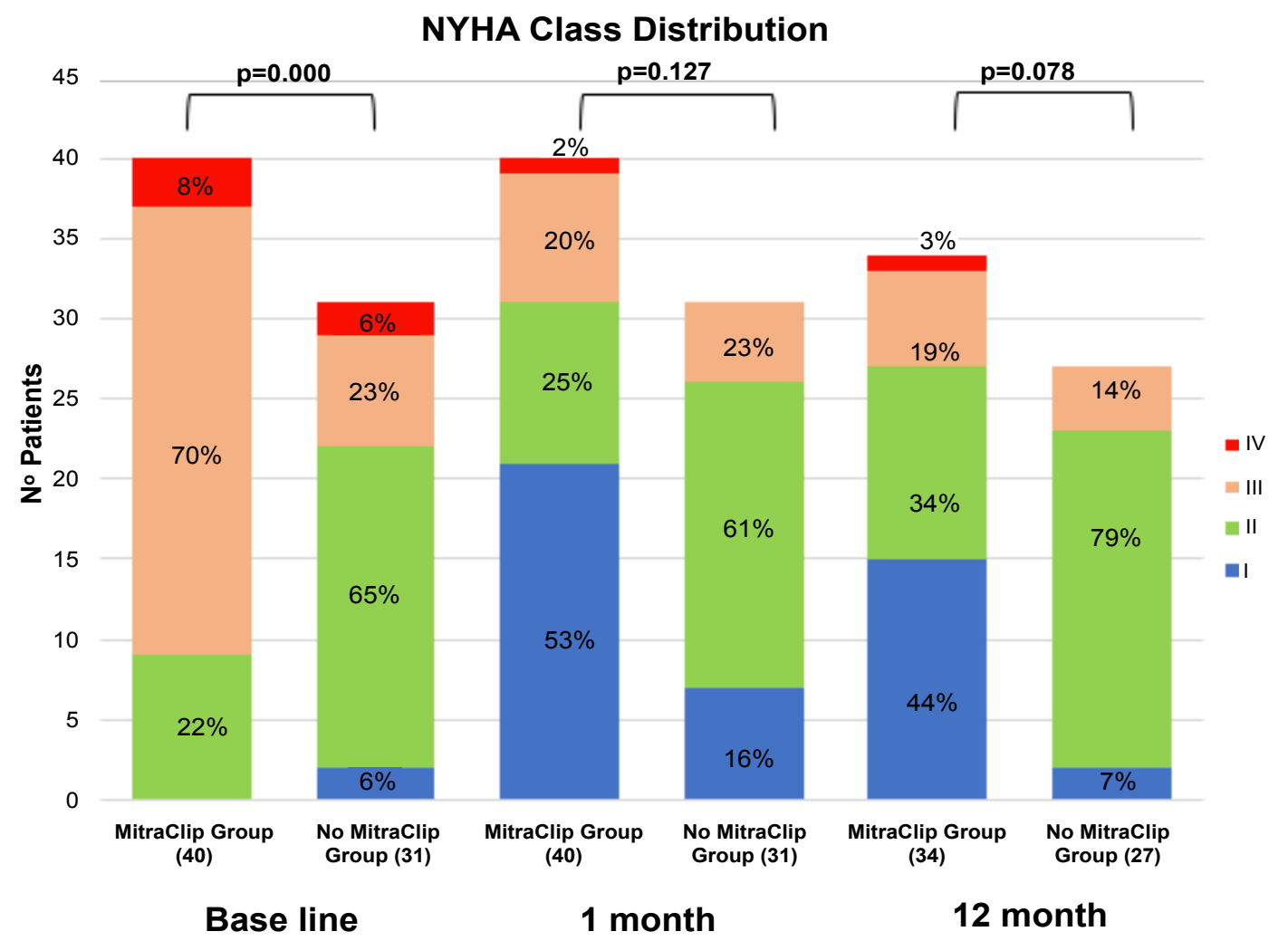

Figure 2: NYHA class distribution in the MitraClip Group and in the no-MitraClip Group at baseline, 30-day and 12-month of follow-up.

30-day follow-up data were available for the overall population (100\%). Death did not occur in anyone of the patients during the first month of follow-up. Conversely, hospitalizations for HF occurred in 6 patients (overall 8.4\%; 2 patients in the MitraClip group and 4 in noMitraClip group). No differences were noted in the NYHA class between the two groups ( $p$-value 0.127; Figure 2). Moreover, no overstated modifications on LVEF were noticed during the follow-up, if compared to baseline (MitraClip group median LVEF $25 \%$ versus noMitraClip group median LVEF 30\%, p-value 0.033 ).

\section{2-month outcome}

Data from 61 patients alive (86\%) were available at 12-month follow-up. As shown in Table 3, there was no statistically significant difference in mortality between the two groups of study: 6 patients (15\%) in
MitraClip group vs. 4 patients (12.9\%) in no-MitraClip group ( $p$-value 0.801 ). Hospitalizations for HF occurred in $23 \%$ of MitraClip patients vs. $32 \%$ of no-MitraClip patients ( $p$-value 0.36). Furthermore, there were no differences in term of NHYA class between MitraClip and no-MitraClip patients ( $p$-value 0.078 ), although, in MitraClip group, paired evaluation in 34 patients alive at 12-month follow-up showed a significant improvement of NHYA class from baseline to 12-month ( $p$-value $\leq$ 0.001 ), as shown in Table 4.

Paired echocardiographic assessment in patients with complete echocardiographic data available at 12-month follow-up showed that LVEF, left ventricle end-diastolic volume and left ventricle end-systolic volume did not significantly differ from baseline to 12-month follow up (Table 4). 
Table 3: Follow-up at 30 days and 12-month.

\begin{tabular}{|c|c|c|c|c|}
\hline 30-day & Overall & MitraClip Group $(n=40)$ & No-MitraClip Group $(n=31)$ & $p$ value \\
\hline Primary Endpoint, $n(\%)$ & $6(8.5 \%)$ & $2(5 \%)$ & $4(12.9 \%)$ & 0.393 \\
\hline Death, n (\%) & $0(0 \%)$ & $0(0 \%)$ & $0(0 \%)$ & - \\
\hline HF Hospitalization, n (\%) & $6(8.5 \%)$ & $2(5 \%)$ & $4(12.9 \%)$ & 0.393 \\
\hline NHYA Class & $2(1-2)$ & $1(1-2)$ & $2(2-3)$ & 0.127 \\
\hline NYHA III-IV, n (\%) & $14(19.7 \%)$ & $9(22.5 \%)$ & $5(16.1 \%)$ & 0.561 \\
\hline LVEF (\%) & $28(24-35)$ & $25(21-32)$ & $30(25-36)$ & 0.033 \\
\hline EDV-LV (ml) & $193(142-247)$ & $210(145-269)$ & $180(113-230)$ & 0.071 \\
\hline ESV-LV (ml) & $130(91-180)$ & $155(94-200)$ & $110(80-160)$ & 0.070 \\
\hline $\mathrm{sPAP}(\mathrm{mmHg})$ & $40(30-50)$ & $40(35-50)$ & $32(25-40)$ & 0.007 \\
\hline Mild-Moderate TR, n (\%) & $55(78.6 \%)$ & $33(82.5 \%)$ & $22(73.3 \%)$ & 0.355 \\
\hline 12-month & Overall $(n=61)$ & MitraClip Group $(n=34)$ & No MitraClip Group ( $n=27$ ) & p-value \\
\hline Primary Endpoint, $n(\%)$ & $27(38 \%)$ & $12(30 \%)$ & $10(32.3 \%)$ & 0.838 \\
\hline Death, n (\%) & $10(14.1 \%)$ & $6(15 \%)$ & $4(12.9 \%)$ & 0.801 \\
\hline HF Hospitalizations, n (\%) & $19(31 \%)$ & $9(22.5 \%)$ & $10(32.3 \%)$ & 0.357 \\
\hline NYHA Class & $2(1-2)$ & $2(1-2)$ & $2(1-2)$ & 0.078 \\
\hline NYHA III-IV, n (\%) & $11(18.3 \%)$ & $7(20.6 \%)$ & $4(14.8 \%)$ & 0.560 \\
\hline LVEF (\%) & $30(25-39)$ & $28(24-35)$ & $33(25-40)$ & 0.129 \\
\hline EDV-LV (ml) & $182(130-233)$ & $196(142-247)$ & $150(142-247)$ & 0.058 \\
\hline ESV-LV (ml) & $125(83-175)$ & $143(93-177)$ & $100(75-169)$ & 0.160 \\
\hline Mild-moderate TR, n (\%) & $53(87 \%)$ & $29(85.3 \%)$ & $24(88.8 \%)$ & 0.680 \\
\hline PAPs $(\mathrm{mmHg})$ & $37(32-45)$ & $35(31-45)$ & $37(31-45)$ & 0.856 \\
\hline
\end{tabular}

Categorical variables are expressed as $\mathrm{n}(\%)$. Continuous variables are expressed as mean $\pm \mathrm{SD}$ and in case of skewed distribution has been reported median and IQR. Primary Endpoint, Death and HF Hospitalizations are counted on the total population (71 patients).

Abbreviations: Primary Endpoint (Composite of death + HF hospitalization); HF: Heart Failure. Other abbreviations as in Table 1.

Table 4: Paired sample T-Test between 34 patients alive in MitraClip group and 27 alive in no-MitraClip group at 12-month follow up: Clinical and echocardiographic outcome.

\begin{tabular}{|c|c|c|c|}
\hline & Baseline & 12-month & p value \\
\hline NYHA Class MitraClip Group & $2.8 \pm 0.5$ & $1.8 \pm 0.8$ & $\leq 0.001$ \\
\hline NYHA Class No-MitraClip group & $2.3 \pm 0.7$ & $2.1 \pm 0.5$ & 0.083 \\
\hline LVEF (\%) MitraClip Group & $30 \pm 9$ & $30 \pm 9$ & 0.533 \\
\hline LVEF (\%) No-MitraClip Group & $32 \pm 7$ & $33 \pm 8$ & 0.464 \\
\hline EDV-LV (ml) MitraClip Group & $206 \pm 67$ & $201 \pm 71$ & 0.364 \\
\hline EDV-LV (ml) No-MitraClip Group & $173 \pm 58$ & $170 \pm 70$ & 0.795 \\
\hline ESV-LV (ml) MitraClip Group & $149 \pm 59$ & $140 \pm 60$ & 0.060 \\
\hline ESV-LV (ml) No-MitraClip Group & $117 \pm 48$ & $119 \pm 55$ & 0.731 \\
\hline
\end{tabular}

Variables are expressed as mean $\pm \mathrm{SD}$.

Abbreviations: Abbreviations as in Table 1.

\section{4-month follow-up}

Kaplan-Meier freedom from all-cause death is depicted in Figure 3A. As shown, no differences between the two groups of study at 24-month were recorded (log rank $p$-value 0.825 ). Figure 3B confirms that the no-MitraClip group presented an increased risk of hospitalizations for HF (log rank p-value 0.048). Moreover, there was not significant difference regarding the primary endpoint at 24-month (Figure 3C; $p$-value $0.32 \mathrm{ss} 5)$.
After adjustment for predefined covariates (including chronic kidney disease, NYHA class and male sex), the incidence of hospital stay for HF or death was significantly delayed in MitraClip group (HR: 0.38; $p$-value 0.018), confirming that chronic kidney disease, NYHA class and male sex were all independent predictors of long-term outcomes (Figure 4).

\section{Discussion}

The present study aimed to evaluate the long-term 

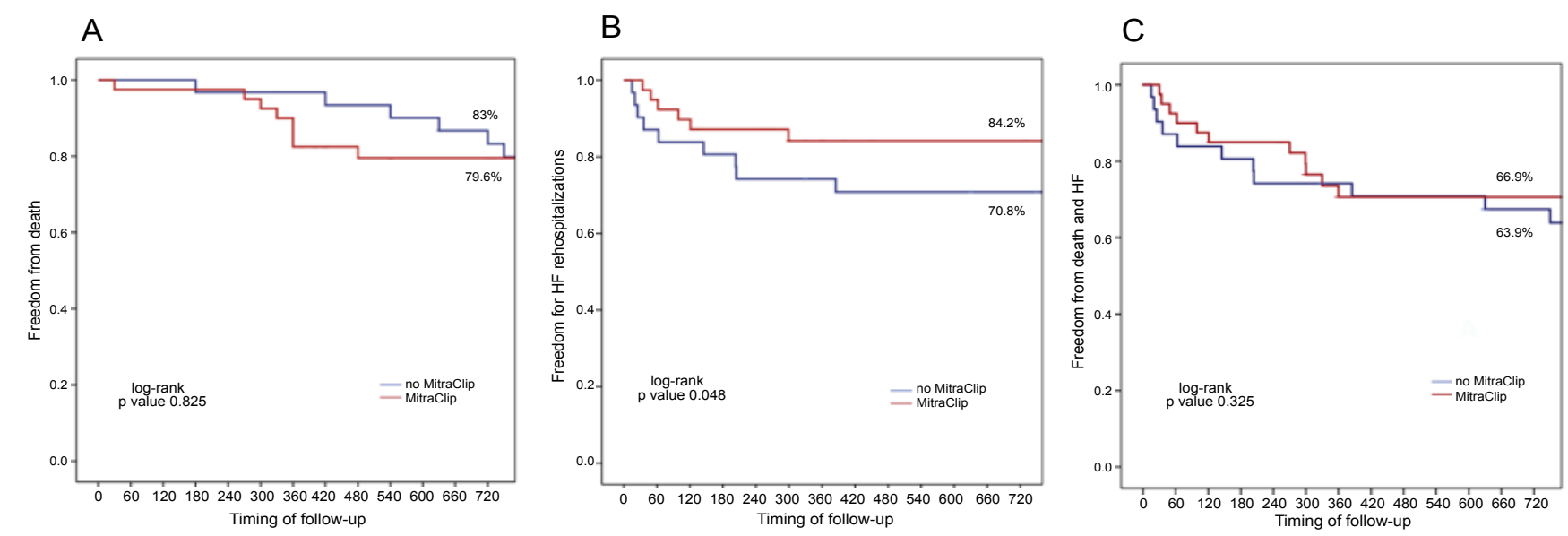

Figure 3: Kaplan Maier curves: A) 24-month freedom from all-cause death; B) 24-month freedom from HF hospitalizations; C) 24-month freedom from the composite of death and HF (primary endpoint). HF: Heart Failure.

\section{MULTIVARIABLE COX REGRESSION ANALYSES OF PRIMARY ENDPOINT (DEATH+HF HOSPITALIZATION)}

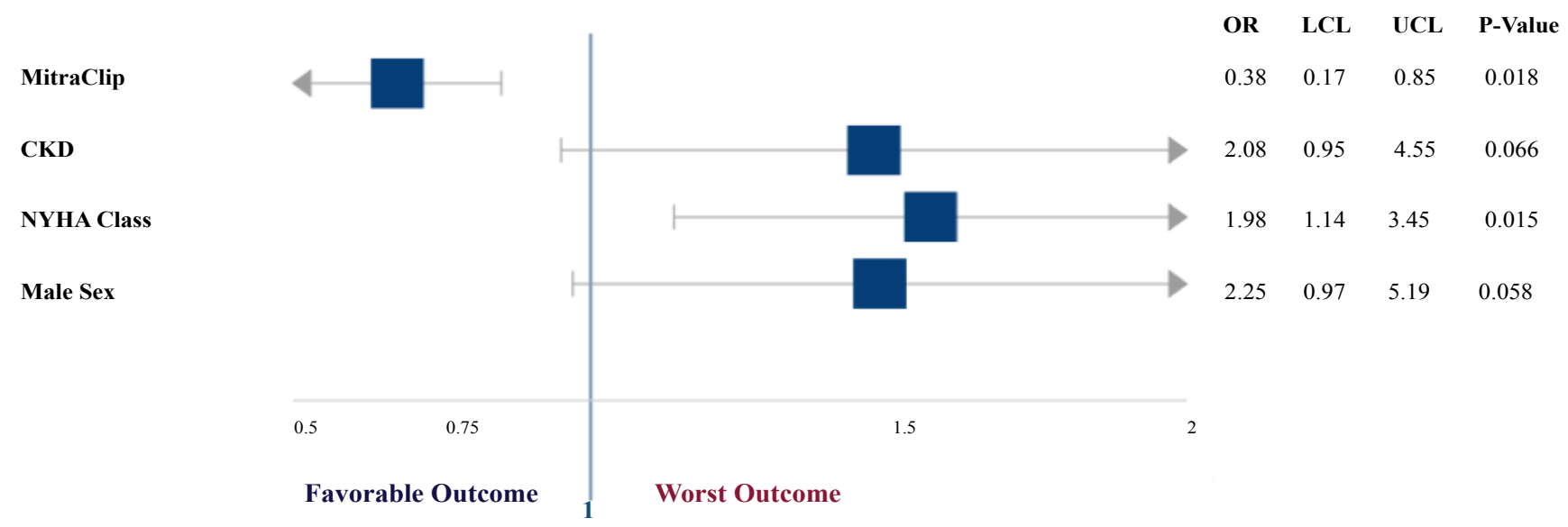

Figure 4: Forest Plot of the multivariable Cox regression analysis identifying covariates of prognostic impact for primary endpoint (composite of death and heart failure hospitalizations), on an epidemiological basis. CKD: Chronic Kidney Disease; OR: Odds Ratio; LCL: Lower Confidence Limit; UCL: Upper Confidence Limit.

outcome (i.e. 24-month) of symptomatic patients with CRT with residual moderate-to-severe FMR who were treated with the MitraClip system or managed with medical therapy alone in a single-center experience. The main finding of this analysis showed that patients treated with the MitraClip System presented lower rate of adverse events (i.e. hospitalization for HF) during a limited period of follow-up, if compared to no-MitraClip patients. Moreover, at 12-month follow-up, the MitraClip group presented a significant improvement of NYHA functional class from baseline. After statistical adjustment for the main epidemiological factors that impact on prognosis, the MitraClip therapy resulted a protective factor against the primary endpoint.

Patients with severely reduced LVEF and FMR are the main focuses of this analysis. FMR is a condition extremely frequent in patients with impaired LVEF, due to the adverse left ventricle remodeling and consequent tenting forces on mitral valve, which are responsible for insufficiency $[16,17]$. Higher degree of FMR after CRT is a strong predictor of all-cause death and hospitalization for $\mathrm{HF}$ at 3 years [18].

In this context it has been demonstrated that MitraClip System in CRT non-responders is a feasible and safe approach and it has showed to increase LVEF, reducing left ventricular volumes [10], with favorable remodeling at mid-term follow-up [11], and to reduce NT-pro BNP levels and tricuspid regurgitation pressure gradient in HF conditions [19]. Two recent trial compared transcatheter mitral-valve repair with medical therapy alone in symptomatic patients with HF with opposite results: In the COAPT study the transcatheter mitral-valve repair compared to medical therapy resulted in a lower rate of all-cause deaths and HF hospitalization within 24 months of follow-up, while MITRA-FR investigators showed no differences in death and HF hospitalization between the two group at one year $[8,9]$. 
Based on these evidences, in our analysis, we sought to evaluate the impact of MitraClip versus medical therapy alone on the outcome of a cohort of patients previously treated with CRT and with residual moderateto-severe FMR in a real-world setting. As per protocol of our Institute and according to the current guidelines, all the CRT patients recruited retrospectively for this study received the maximum tolerated dose of medical therapy for HF. Apart from the patients who died before the introduction of the MitraClip at our Institute and were managed only with medical therapy, all the patients were proposed for the MitraClip procedure and this intervention was denied just in case of absolute or relative contraindications or refusal of the patient.

From this initial analysis, we interestingly noticed that, at baseline, the MitraClip group did not typically present the favorable demographic characteristics of the CRT responder population. Indeed, in the MitraClip group we can register a higher prevalence of men with history of ischemic cardiomyopathy, whereas female sex and non-ischemic cardiomyopathy generally showed an increased responsiveness to CRT. If we also add that the MitraClip group presents a higher surgical risk profile according to the comorbidities presented and advanced symptoms in terms of higher NYHA class, it's interesting to notice that the MitraClip group showed a more favorable outcome in term of hospitalizations for $\mathrm{HF}$ during the follow-up, if compared to the no-MitraClip group, although no differences in primary endpoint and all-cause mortality were detected between the two groups at 24-month.

As regards the peri-procedural outcome of the MitraClip group, all the clips were deployed with low rate of complications (7.5\%) and the technical success in these patients was in line with the results of the GRASP registry, which were previously published [20]. All the procedural complications were promptly managed and no in-hospital deaths were reported. The device time in the MitraClip group (i.e. median 49 minutes, IQR 29-75) was similar to the updated device time of the overall population of the GRASP registry (i.e. median 51 minutes, IQR 30-75), showing that the presence of an electronic device such as CRT does not impact on the technical aspect of the procedure itself. Another interesting point is that the MitraClip group required a larger number of clips (i.e. median 2) if compared to the overall population of the GRASP registry (i.e. median 1) [20]. A possible explanation of this finding could be that, patients with larger ventricle volumes, more sever annular dilatation and increased tethering forces leading to malcoaptation of the scallops require more clips to get a satisfactory result, if compared to FMR with better left ventricle function or degenerative mitral regurgitation.

Despite the small number of patients recruited in this study, our results from real clinical practice confirm the feasibility and effectiveness of the MitraClip procedure in a cohort of high-risk patients with severe left ventricle dysfunction, suggesting that when appropriate, according to the anatomical and clinical features, this procedure should be routinely proposed.

The favorable results of the MitraClip in term of improvement of NYHA functional class and MR reduction remained sustained during the 30-day and 12-month follow-up, and the difference in term of left ventricle volumes and left ventricle dysfunction, that were statistically significant between MitraClip group and no-MitraClip group before the MitraClip procedure, were smoothed. The incidence of death at 30-day and 12-month was low and not statistically significant between the two groups of study. During the follow-up, the MitraClip group presented a significant improvement in NYHA functional class and a mild, but not significant, favorable left ventricle reverse remodeling, with a leveling in the echocardiographic differences between the two study groups from baseline to the 12-month follow-up. Reverse left ventricle remodeling after CRT and MitraClip has been described in literature $[10,11]$ and this event seems to be related to a better outcome during the follow-up.

Another critical result is that the MitraClip group, if compared to no-MitraClip group, showed a lower rate of hospitalizations for HF in a 24-month KaplanMeier analysis and the Cox Proportional-Hazards Model strengthened this evidence, showing that MitraClip was protective against the primary composite endpoint.

These results are in line with studies that show that MitraClip helps to reduce the hospitalizations rate for HF and improve quality of life $[8,20,21]$. Frequent hospitalizations are associated with a worse long-term outcome and significant increase in HF-related health costs. Furthermore, it was observed that at every hospital readmission, the quality of life of patients declines [22].

\section{Limits}

Of course, several limitations should be considered inherent to our study, by the virtue of its observational nature. This single-center study included a small number of patients and there are significantly baseline difference between the two groups compared. So the results should not be generalized and further, larger multicenter studies are needed to confirm these exploratory results.

\section{Conclusion}

In CRT patients, the treatment of moderate-tosevere FMR with the Mitraclip System - if compared to medical therapy alone - is a feasible, safe and reasonably effective approach in reducing cardiac symptoms and the rate of hospitalizations for HF. 


\section{Disclosures}

Carmelo Grasso is a proctor physician for Abbott Vascular.

Davide Capodanno received consulting and speaker's honoraria from Abbott Vascular.

Corrado Tamburino received speaker's honoraria from Abbott Vascular.

All other authors have no conflicts of interest to declare.

\section{References}

1. Ponikowski $P$, Voors $A A$, Anker SD, Bueno $H$, Cleland JGF, et al. (2016) 2016 ESC Guidelines for the diagnosis and treatment of acute and chronic heart failure: The Task Force for the diagnosis and treatment of acute and chronic heart failure of the European Society of Cardiology (ESC). Eur Heart J 37: 2129-2200.

2. Di Biase L, Auricchio A, Mohanty P, Bai R, Kautzner J, et al. (2011) Impact of cardiac resynchronization therapy on the severity of mitral regurgitation. Europace 13: 829-838.

3. Sitges M, Vidal B, Delgado V, Mont L, Garcia-Alvarez A, et al. (2009) Long-term effect of cardiac resynchronization therapy on functional mitral valve regurgitation. Am J Cardiol 104: 383-388.

4. Van Bommel RJ, Marsan NA, Delgado V, Borleffs CJ, van Rijnsoever EP, et al. (2011) Cardiac resynchronization therapy as a therapeutic option in patients with moderatesevere functional mitral regurgitation and high operative risk. Circulation 124: 912-919.

5. Baumgartner H, Falk V, Bax JJ, De Bonis M, Hamm C, et al. (2017) 2017 ESC/EACTS Guidelines for the management of valvular heart disease. Eur Heart J 38: 2739-2791.

6. Puls $M$, Lubos E, Boekstegers $P$, von Bardeleben RS, Ouarrak T, et al. (2016) One-year outcomes and predictors of mortality after MitraClip therapy in contemporary clinical practice: Results from the German transcatheter mitral valve interventions registry. Eur Heart J 37: 703-712.

7. Feldman $T$, Kar $S$, Rinaldi $M$, Fail $P$, Hermiller $J$, et al. (2009) Percutaneous mitral repair with the MitraClip system: Safety and midterm durability in the initial EVEREST (Endovascular Valve Edge-to-Edge Repair Study) cohort. J Am Coll Cardiol 54: 686-694.

8. Stone GW, Lindenfeld J, Abraham WT, Kar S, Lim DS, et al. (2018) Transcatheter Mitral-Valve Repair in Patients with Heart Failure. N Engl J Med 379: 2307-2318.

9. Obadia JF, Messika-Zeitoun D, Leurent G, lung B, Bonnet G, et al. (2018) Percutaneous Repair or Medical Treat-ment for Secondary Mitral Regurgitation. N Engl J Med 379: 2297-2306.

10. Auricchio A, Schillinger W, Meyer S, Maisano F, Hoffmann $R$, et al. (2011) Correction of mitral regurgitation in nonresponders to cardiac resynchronization therapy by MitraClip improves symptoms and promotes reverse remodeling. J Am Coll Cardiol 58: 2183-2189.
11. Giaimo VL, Zappulla P, Cirasa A, Tempio D, Sanfilippo $M$, et al. (2018) Long-term clinical and echocardiographic outcomes of Mitraclip therapy in patients non responders to cardiac resynchronization. Pacing Clin Electrophysiol 41: 65-72.

12. Stone GW, Vahanian AS, Adams DH, Abraham WT, Borer JS, et al. (2015) Clinical trial design principles and endpoint definitions for transcatheter mitral valve repair and replacement: Part 1: Clinical trial design principles: A consensus document from the mitral valve academic research consortium. J Am Coll Cardiol 66: 278-307.

13. Lancellotti P, Tribouilloy C, Hagendorff A, Popescu BA, Edvardsen T, et al. (2013) Recommendations for the echocardiographic assessment of native valvular regurgitation: An executive summary from the European Association of Cardiovascular Imaging. Eur Heart J Cardiovasc Imaging 14: 611-644.

14. Lang RM, Badano LP, Mor-Avi V, Afilalo J, Armstrong A, et al. (2015) Recommendations for cardiac chamber quantification by echocardiography in adults: An update from the American Society of Echocardiography and the European Association of Cardiovascular Imaging. J Am Soc Echocardiogr 28: 1-39.

15. Fornwalt BK, Sprague WW, Be Dell P, Suever JD, Gerritse $B$, et al. (2010) Agreement is poor among current criteria used to define response to cardiac resynchronization therapy. Circulation 121: 1985-1991.

16. Asgar AW, Mack MJ, Stone GW (2015) Secondary mitral regurgitation in heart failure: Pathophysiology, prognosis, and therapeutic considerations. J Am Coll Cardiol 65: 12311248.

17. Rossi A, Dini FL, Faggiano P, Agricola E, Cicoira M, et al. (2011) Independent prognostic value of functional mitral regurgitation in patients with heart failure. A quantitative analysis of 1256 patients with ischaemic and non-ischaemic dilated cardiomyopathy. Heart 97: 1675-1680.

18. Upadhyay GA, Chatterjee NA, Kandala J, Friedman DJ, Park MY, et al. (2015) Assessing mitral regurgitation in the prediction of clinical outcome after cardiac resynchronization therapy. Heart Rhythm 12: 1201-1208.

19. Seifert M, Schau T, Schoepp M, Arya A, Neuss M, et al. (2014) MitraClip in CRT non-responders with severe mitral regurgitation. Int J Cardiol 177: 79-85.

20. Grasso C, Popolo Rubbio A, Capodanno D, Buccheri S, Di Salvo ME, et al. (2018) Incidence, timing, causes and predictors of early and late re-hospitalization in patients who underwent percutaneous mitral valve repair with the mitraclip system. Am J Cardiol 121: 1253-1259.

21. Vakil K, Roukoz H, Sarraf M, Krishnan B, Reisman M, et al. (2014) Safety and efficacy of the MitraClip system for severe mitral regurgitation: A systematic review. Catheter Cardiovasc Interv 84: 129-136.

22. Nieminem MS, Dickstein K, Fonseca C, Serrano JM, Parissis J, et al. (2015) The patient perspective: Quality of life in advanced heart failure with frequent hospitalizations. Int J Cardiol 191: 256-264. 